\title{
Acidente por picada de abelha africanizada em dois equinos
}

\author{
Africanized bee sting accident in two horses
}

\author{
Joana Cristina Smaha de Jesus Lima (iD ${ }^{1 *}$, Monique Togni Martins (D)2 ${ }^{2}$ Francisco Augusto Ricci Catalano (iD ${ }^{3,4}$ \\ 1 Programa de Pós-Graduação em Ciência Animal, Universidade Federal do Paraná (UFPR), Palotina, PR, Brasil \\ 2 Universidade Regional Integrada do Alto Uruguai e das Missões (URI), Santiago, RS, Brasil \\ ${ }^{3}$ Centro Universitário Integrado (CEI), Campo Mourão, PR, Brasil \\ ${ }^{4}$ Instituto Integrado de Ciência e Tecnologia (IN2), Campo Mourão, PR, Brasil
}

\section{Resumo}

O presente trabalho tem como objetivo descrever as alterações clínicas, anatomopatológicas e tratamento de dois equinos que sofreram múltiplas ferroadas de abelhas africanizadas. Duas éguas, uma Quarto de Milha (pelagem Baio Amarilho) e outra mestiça (pelagem Tobiano), ambas pesando aproximadamente $400 \mathrm{~kg}$, sofreram um ataque por abelhas na área rural da cidade de Campo Mourão, Paraná, sul do Brasil. Os animais apresentavam múltiplos ferrões distribuídos pelo corpo, ambos tiveram edema de face, salivação, dispneia, incoordenação motora, nistagmo (égua tobiano) e mudanças no nível de consciência. No tratamento inicial foi realizada fluidoterapia com solução de ringer lactato e administrado prometazina, dexametazona e dimetilsulfóxido. As éguas responderam ao tratamento inicial revertendo o quadro neurológico cinco horas

* Autor correspondente: joanacristinasmaha@gmail.com Submetido: 25 ago 2020 | Aprovado: 11 set 2020 após o início do tratamento, porém a égua mais ferroada veio a óbito em 12 horas. Na necropsia, o fígado ao corte apresentava coloração amarelo dourada e o rim estava acentuadamente vermelho escuro com pontos enegrecidos no córtex renal. A laringe apresentava edema difuso acentuado de glote. $O$ encéfalo apresentava avermelhamento difuso acentuado com achatamento dorso ventral das circunvoluções. No exame histopatológico, as lesões mais marcantes foram nos rins. $O$ diagnóstico de crise hemolítica de origem tóxica e de intoxicação histamínica de ambos os animais foi baseado nos ferrões encontrados na pele dos animais, associados aos sinais clínicos e achados post mortem no equino que veio a óbito.

Palavras-chave: Cavalo. Intoxicação histamínica. Apitoxina. 


\section{Abstract}

The present work aims to describe the clinical, anatomopathological and treatment changes of two horses that suffered multiple stings from Africanized bees. Two mares, one Quarter Horse and the other mixed breed, both weighing approximately $400 \mathrm{~kg}$, were attacked by bees in the rural area of the city of Campo Mourão, Paraná, south of Brazil. The animals had multiple stingers distributed throughout the body, both had edema of the face, salivation, dyspnea, motor incoordination, nystagmus (tobiano mare) and changes in the level of consciousness. In the initial treatment, fluid therapy with ringer lactate and promethazine, dexamethasone and dimethylsulfoxide, were administered. The mares responded to the initial treatment by reversing the neurological condition five hours after the start of treatment, but the most stung mare died within 12 hours. At necropsy, the liver at the cut had a golden yellow color and the kidney was markedly dark red with blackened spots in the renal cortex. The larynx had marked diffuse edema of the glottis. The brain had diffuse redness accentuated with flattened ventral dorsum of the circumvolutions. In histopathology, the most striking lesions were in the kidney. The diagnosis of hemolytic crisis of toxic origin and histamine poisoning of both animals were based on the stings found on the animals' skin, associated with clinical signs, and post-mortem findings in the horse that died.

Keywords: Horse. Histaminic intoxication. Whistle.

\section{Introdução}

Os acidentes causados por múltiplas picadas de abelhas normalmente são bastante graves, sendo geralmente fatais em animais domésticos devido à indução de graves reações tóxicas e alérgicas causada pela apitoxina (Sakate, 2008). A composição da apitoxina ou o volume de uma única picada não são importantes em indivíduos não alérgicos, porém a dose acumulativa de várias picadas contribui para a mortalidade associada a incidentes com abelhas africanizadas (Sherman, 1995). Há três tipos distintos de reações a picadas de abelhas africanizadas. No primeiro, o indivíduo não sensibilizado recebe poucas picadas de abelhas e desenvolve reação inflamatória local. No segundo, o indivíduo já sensibilizado recebe uma picada, desencadeando uma reação de hipersensibilidade. E o último, conhecido como síndrome de envenenamento, ocorre devido a múltiplas picadas, geralmente mais de 500 ferroadas, gerando efeitos tóxicos no organismo (Mendes et al., 1990).

As manifestações clínicas variam, dependendo da quantidade de picadas que o animal recebe e da sua suscetibilidade, podendo ocorrer sinais neurológios, crise hemolítica e rabdomiólise (Oliveira et al., 2007; Sakate, 2008). A hemoglobinúria e/ou mioglobinúria desenvolvidas causam necrose tubular aguda, levando o animal à morte por insuficiência renal aguda (Fighera et al., 2007).

$O$ presente trabalho tem como objetivo descrever as alterações clínicas, anatomopatológicas e tratamento de dois equinos que sofreram múltiplas ferroadas de abelhas africanizadas.

\section{Relato de caso}

Duas éguas, uma Quarto de Milha de pelagem Baio Amarilho (égua 1) e uma mestiça de pelagem Tobiano (égua 2), ambas pesando aproximadamente $400 \mathrm{~kg}$, sofreram um ataque por abelhas na área rural da cidade de Campo Mourão, Paraná, sul do Brasil. Os animais apresentavam múltiplos ferrões distribuídos pelo corpo, ambos tiveram edema difuso de face (Figura 1), salivação, dispneia, incoordenação motora e mudanças no nível de consciência caracterizado por excitabilidade e o não reconhecimento de outros animais e pessoas a sua volta. Nistagmo foi manifestado apenas na égua 2 . Ao exame físico ambas as éguas apresentavam frequência respiratória entre 10 e 15 movimentos por minuto, frequência cardíaca acima de 80 batimentos por minuto, turgor cutâneo maior que cinco segundos, tempo de preenchimento capilar de quatro segundos, mucosa oral rósea com petéquias, hipotermia e urina de coloração vinho tinto. 
Realizou-se o tratamento dos dois animais, ao qual égua 1 foi responsiva, porém a égua 2, a mais ferroada, apresentou sinais de choque anafilático e veio a óbito em 12 horas. Foi realizada necropsia e os fragmentos foram encaminhados para análise histopatológica no Laboratório de Patologia Animal do Centro Universitário Integrado de Campo Mourão. Os mesmos foram fixados em formalina a $10 \%$ e corados pelo método hematoxilina e eosina.

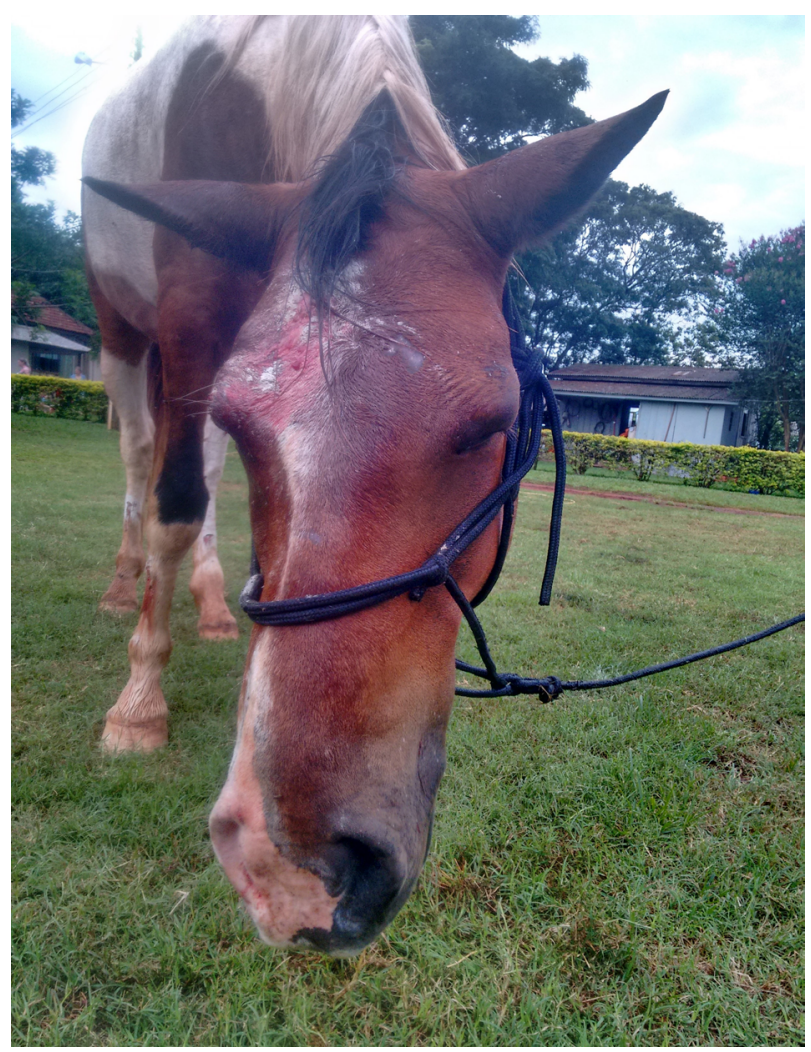

Figura 1 - Equino mestiço (égua 2). Edema difuso de face observado desde a região auricular até os lábios superiores, após acidente por picada de abelhas africanizadas.

\section{Resultados}

No tratamento inicial foi realizada fluidoterapia com 10 litros de ringer lactato (JP Indústria Farmacêutica S.A., Ribeirão Preto/SP) e administrado $0,2 \mathrm{mg} / \mathrm{kg}$ de prometazina (Cristália, Cotia/ SP) I.V., 0,2 $\mathrm{mg} / \mathrm{kg}$ de dexametazona (MSD
Saúde Animal, São Paulo/SP) I.V. e $1 \mathrm{~g} / \mathrm{kg}$ de dimetilsulfóxido (Ceva, Paulínia/SP), diluído a 10\% em cinco litros de solução fisiológica (JP Indústria Farmacêutica S.A., Ribeirão Preto/SP), sendo necessária a administração de 20 microgramas $/ \mathrm{kg}$ de detomidina (Agener União, São Paulo/SP) I.V. para contenção da égua 1.

As éguas responderam ao tratamento inicial, revertendo o quadro neurológico cinco horas após o início do tratamento. Entretanto, a égua 2, a mais ferroada, apresentou sinais de choque anafilático e veio a óbito em 12 horas. A égua 1 progrediu para um estado de apatia, anúria, aumento da área edematosa na face, mucosa oral e vaginal levemente ictéricas e sangramento persistente nos orifícios gerados por agulhas e cateteres. A fluidoterapia com 10 litros de ringer lactato pela manhã e a administração de $0,1 \mathrm{mg} /$ $\mathrm{kg}$ de dexametazona I.V. foram mantidas até o desaparecimento dos sinais clínicos, tendo o animal a recuperação completa em quatro dias.

$\mathrm{Na}$ necropsia da égua 2 as mucosas estavam congestas, a gordura moderadamente amarela (icterícia leve), o fígado ao corte apresentava coloração amarelo dourada e o rim estava acentuadamente vermelho escuro (Figura 2), com pontos enegrecidos no córtex renal.

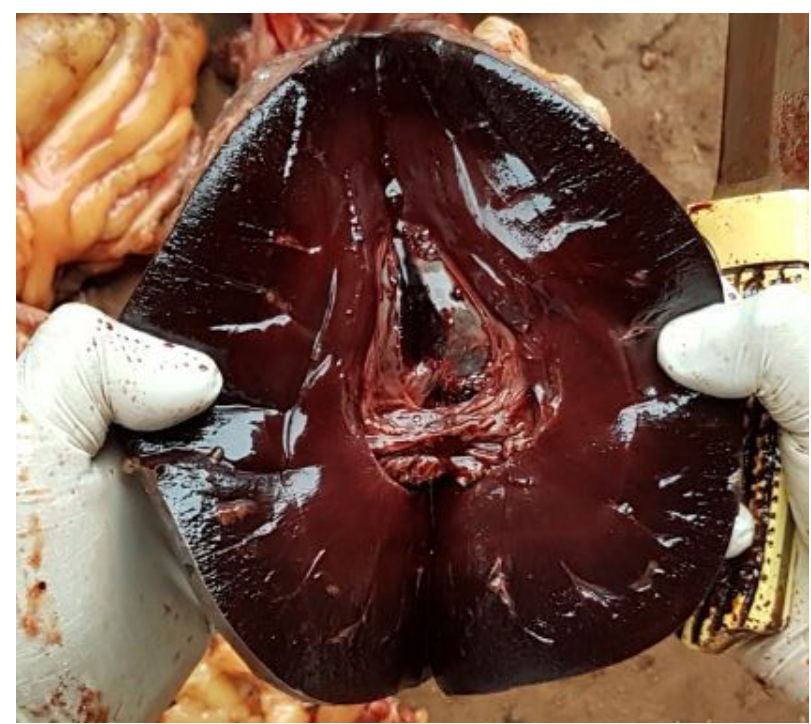

Figura 2 - Rim de um equino mestiço, fêmea (égua 2), com congestão difusa acentuada após acidente por picada de abelhas africanizadas. 
O baço estava acentuadamente aumentado (esplenomegalia), no miocárdio havia áreas pálidas e o músculo papilar do ventrículo direito estava enegrecido. A laringe apresentava edema difuso acentuado de glote e o encéfalo apresentava avermelhamento difuso acentuado com achatamento dorso ventral das circunvoluções (Figura 3).

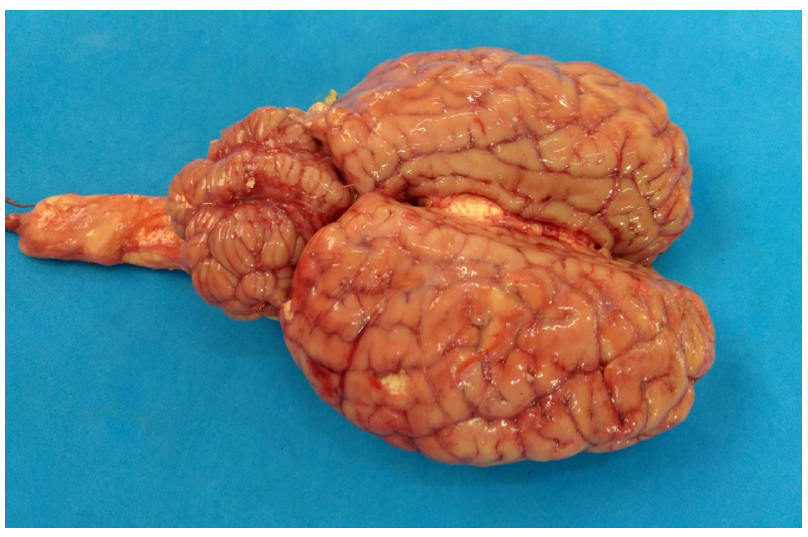

Figura 3 - Encéfalo de um equino mestiço, fêmea (égua 2), com congestão difusa acentuada com achatamento dorso ventral das circunvoluções, após acidente por picada de abelhas africanizadas.

No exame histopatológico, as lesões mais marcantes foram nos rins. As células do epitélio de revestimento tubular apresentavam acentuada degeneração e necrose, grande parte dos túbulos contorcidos distais e proximais estavam preenchidos por material eosinofílico (cilindros de hemoglobina) e alguns túbulos apresentavam debris celulares em sua luz (Figura 4) e congestão vascular difusa acentuada. $O$ diagnóstico morfológico foi necrose tubular aguda associada à presença de cilindros de hemoglobina intratubulares e congestão difusa acentuada.

No encéfalo, principalmente na ponte, observou-se congestão acentuada e edema perineuronal e perivascular, além de vacúolos multifocais no neurópilo (Figura 5). O fígado apresentava congestão difusa moderada com depósitos de hemossiderina em hepatócitos. No coração, no músculo papilar, havia congestão e hemorragia multifocal acentuada com depósito de hemossiderina em cardiomiócitos e macrófagos, sendo essas alterações também encontradas no baço. O pulmão apresentava congestão, hemorragia e edema difuso acentuado.

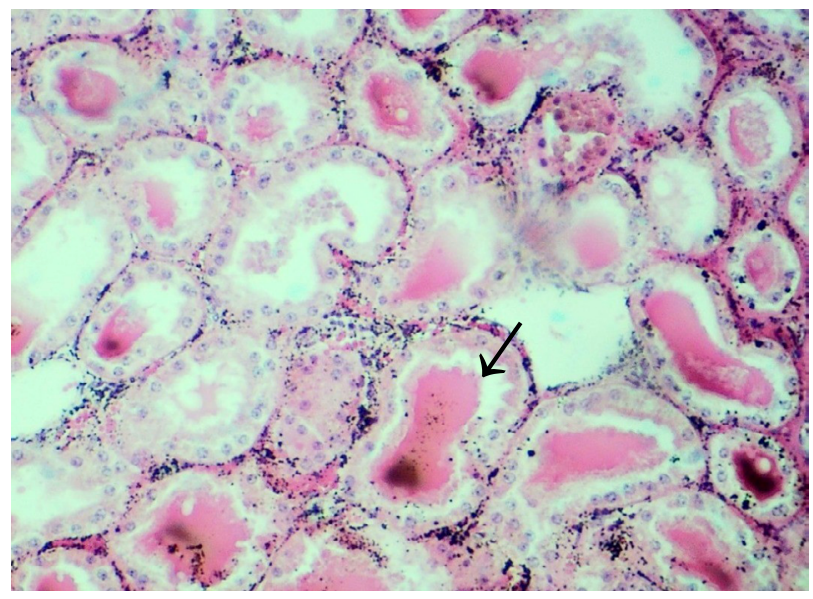

Figura 4 - Corte histológico do rim de um equino mestiço, fêmea (égua 2). Microscopia túbulos contorcidos distais e proximais estavam preenchidos por material eosinofílico (cilindros de hemoglobina) (seta preta) após acidente por picada de abelhas africanizadas. Coloração H\&E. Objetiva de 100x.

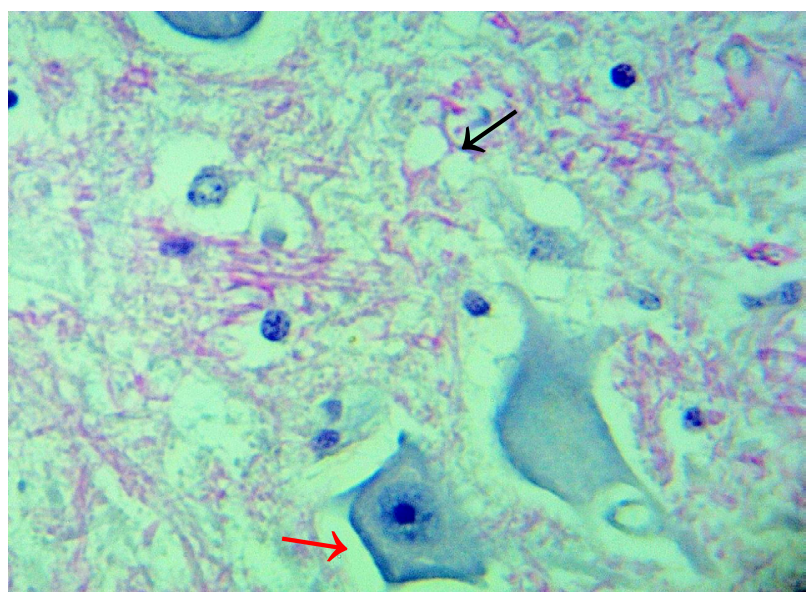

Figura 5 - Corte histológico do encéfalo de um equino mestiço, fêmea (égua 2). Microscopia edema perineuronal (seta vermelha), perivascular além de vacúolos multifocais no neurópilo (seta preta) após acidente por picada de abelhas africanizadas. Coloração H\&E. Objetiva de 100x. 


\section{Discussão}

Os acidentes por picadas de abelhas ocorrem com muita frequência em animais domésticos, principalmente em cães e equinos, mas existem poucos relatos descritos no Brasil nestas espécies (Fighera et al., 2007; Oliveira et al., 2007; Nunes et al., 2017), gerando, assim, um desconhecimento da importância e da patogênese dessa toxicose (Fighera et al., 2007).

A égua 2, de pelagem Tobiano (marrom com manchas brancas) possuía mais ferroadas aparentes do que a égua 1 , de pelagem Baio Amarilho (amarela). Isso corrobora com a literatura, que afirma maior susceptibilidade a picadas de abelhas em animais com pelagem escura do que os de pelagem clara (Sakate, 2008).

Em camundongos, os sinais neurológicos decorrentes de picadas de abelhas foram relacionados com a apamina, constituinte do veneno. Ela tem atuação na junção neuromuscular, resultando em hiperexcitabilidade e convulsão (Habermann, 1972). A melitina, outro constituinte do veneno, pode apresentar ação inibidora da anticolinesterase, ocasionando excitação sináptica (Mendes et al., 1990). Com a ação de ambas as toxinas, o animal pode apresentar excitabilidade entre outros sinais neurológicos, semelhante às éguas deste relato.

Os distúrbios hemolíticos são uma das principais causas de insuficiência renal aguda (Nunes et al., 2010) e, em cães, estes ditúrbios são as principais complicações dos acidentes por picadas de abelhas (Fighera et al., 2007). As lesões renais macro e microscópicas associadas às alterações em outros órgãos e tecidos, como os ferrões na pele, icterícia, esplenomegalia e hemossiderose em múltiplos órgãos, confirmam os casos em questão se tratarem de crise hemolítica tóxica, sendo a necrose tubular aguda consequência desta. Apesar de não ter sido dosada a creatinina sérica da égua 1, esta apresentou quadro de anúria por 12 horas, caracterizando insuficiência renal aguda (Fonteque et al., 2015).

Alterações renais semelhantes podem ser resultantes da mioglobinuria após a destruição de grande quantidade de miofibras (Oliveira et al., 2007; Daher et al.,2009; Nunes et al., 2010); porém, não sendo observadas alterações em miofibras, esta hipótese foi descartada. Acreditase que alguns constituintes presentes no veneno da abelha possam ter ações tóxicas diretas nas células do epitélio tubular renal (Reis et al.,1998).

Os sinais clínicos como edema de face, dispneia e vasodilatação periférica, observados na égua 1, bem como edema pulmonar, edema de glote e congestão, encontrados na égua 2, são decorrentes principalmente da ação vasoativa da histamina e dos leucotrienos liberados endogenamente devido a outros componentes do veneno, como a fosfolipase A2 e o peptídeo desgranulador de mastócitos (Habermann, 1972; Fonteque et al., 2015). Essas alterações são descritas em humanos como intoxicação histamínica (Vetter et al., 1999).

Para o tratamento de múltiplas picadas de abelhas não há antiveneno específico disponível para animais (Sakate, 2008), mas sabe-se que este soro já foi desenvolvido por pesquisadores brasileiros e está em fase clínica de testes (Terças et al., 2017). Conforme os sinais clínicos apresentados, o animal deve receber cuidados de suporte e monitoração constante. A prometazina e dexametazona são indicadas por apresentarem ação anti-histamínica (Gomes, 2008). O dimetilsulfóxido, além de potencializador da dexametazona, apresenta ação antiedematosa; a ativação dos alfa 2-adrenorreceptores présinápticos, causada pela ação da detomidina, pode bloquear a entrada de cálcio no terminal nervoso (Bagatini et al.,2002), reduzindo a ação excitatória do veneno. A terapia com fluído é necessária para aumentar a taxa de filtração, uma vez que o animal esteja em anúria (Fighera et al., 2007).

O prognóstico está relacionado à quantidade de picadas por $\mathrm{kg}$ de peso. Normalmente, animais acima de 24 picadas de abelha por $\mathrm{kg}$ vem a óbito (Sakate, 2008).

\section{Conclusão}

Os sinais clínicos como excitabilidade, edema e anúria, bem como os achados post mortem de necrose tubular renal com presença de cilindros de hemoglobina, esplenomegalia e hemossiderose são importantes para o diagnóstico de acidentes 
por picadas de abelhas. Todos os sinais clínicos e alterações post mortem desses animais indicaram crise hemolítica e intoxicação por histamina, que associados aos ferrões na pele comprovam tratarse de acidente por picadas de abelhas. Sabe-se que alguns constituintes do veneno das abelhas, como melitina, fosfolipase A2 e peptídeo desgranulador de mastócitos, são os responsáveis pelos sinais clínicos e lesões post mortem apresentadas pelas éguas deste relato.

\section{Referências}

Bagatini A, Gomes CR, Masella MZ, Rezer G. Dexmedetomidina: farmacologia e uso clínico. Rev Bras Anestesiol. 2002;52(5):606-17.

Daher EF, Oliveira RA, Silva LSV, Bezerra e Silva EM, Morais TP. Insuficiência renal aguda por picada de abelhas: relato de casos. Rev Soc Bras Med Trop. 2009;42(2):209-12.

Fighera RA, Souza TM, Barros CSL. Acidente provocado por picada de abelhas como causa de morte de cães. Cienc Rural. 2007;37(2):590-3.

Fonteque JH, Mendes RP, Souza AF, Granella MCS, Yonezawa LA, Volpato J. Acidente por picada de abelha em equino. $42^{\circ}$ Congresso Brasileiro de Medicina Veterinária e $1^{\circ}$ Congresso Sul-Brasileiro da ANCLIVEPA; 31 out - 2 nov 2015; Curitiba, PR.

Gomes SGR. Hemocomponentes e principais aplicações na terapia intensiva veterinária. In: Santos MM, Fragata FS. Emergência e terapia intensiva veterinária em pequenos animais: Bases para o atendimento hospitalar. São Paulo: Roca; 2008. p. 191-207.
Habermann E. Bee and wasp venoms. Science. 1972; 177(4046):314-22.

Mendes RP, Meira DA, Molinari H, Rodrigues PS, Coelho KYR. Acidentes por múltiplas picadas de abelha. Arq Bras Med. 1990;64:81-8.

Nunes NJS, Matheus JP, Rocha MB, Ribes AO, Machado $L$, Duda NCB, et al. Síndrome do envenenamento por picada de abelha em cão. Investigação. 2017;16 (3).

Nunes TF, Brunetta DM, Leal CM, Pisi PCB, Roriz-Filho JS. Insuficiência renal aguda. Medicina (Ribeirão Preto). 2010;43(3):272-82.

Oliveira EC, Pedroso PMO, Meirelles AEWB, Pescador CA, Gouvêa AS, Driemeier D. Pathological findings in dogs after multiple Africanized beestings. Toxicon. 2007;49(8): 1214-8.

Reis MA, Costa RS, Coimbra TM, Teixeira VP. Acute renal failure in experimental envenomation with Africanized bee venom. Ren Fail. 1998;20(1):39-51.

Sakate M. Zootoxinas. In: Spinosa HS, Górniac SL, PalermoNeto J. Toxicologia aplicada à medicina veterinária. São Paulo: Manole; 2008. p. 209-55.

Sherman RA. What physicians should know about Africanized honeybees. West J Med. 1995;163(6):541-6.

Terças ACP, Vivi VK, Machado C, Lemos ERS. Aspectos epidemiológicos dos acidentes por picada de abelha africana. J Health NPEPS. 2017;2(Supl 1):58-72.

Vetter RS, Visscher PK, Camazine S. Mass envenomations by honey bees and wasps. West J Med. 1999;170(4):223-7. 